\title{
TREATMENT OF SUPERFICIAL OCULAR INFECTION WITH A POLYMYXIN-BACITRACIN OINTMENT (POLYFAX)* +
}

\author{
BY \\ D. AINSLIE \\ Institute of Ophthalmology, University of London
}

SUPERFICIAL ocular infections, for example blepharitis, are better treated by local applications than by systemic therapy. Local applications of antibiotic and chemotherapeutic agents are also of value in reducing the incidence of infection after corneal injury. Sulphacetamide is most commonly used but, although its value in the prevention of corneal infection is well established (Cameron, 1949), it is active only against a limited range of organisms. Ps. pyocyaneus in particular tends to be resistant to sulphacetamide. Penicillin too is of limited value, being effective only against Gram-positive and coccal organisms, and liable to induce allergic responses. Chloromycetin, aureomycin, and terramycin fail to provide full cover against Ps. pyocyaneus.

In the treatment of infection the ideal procedure is to discover the causative organism by bacteriological examination, so that the most suitable antibiotic may be chosen. This ideal is, however, seldom possible in practice outside hospital, and a topical application active against a large range of organisms and likely to be effective when used empirically is desirable. Indeed, in all cases treatment will have to be started without bacteriological confirmation of the type of infection as it is not justifiable to delay while awaiting the result of culture. Further, the wider the range of antibacterial activity, the greater the value as a prophylactic against wound infection.

The perfect antibiotic preparation would be lethal to all ocular pathogens, and sensitive organisms would not be able to develop resistance. In addition a topical application must be non-irritant when applied to the eye and must not give rise to allergic reactions. It is not yet possible to fulfill all these conditions, but the antibiotics bacitracin and polymyxin used together satisfy most of them.

Bacitracin and polymyxin are both polypeptides and are extracted from products of growth of the B. licheniformis and $B$. polymyxa respectively (Finland and Wright, 1953). Bacitracin is effective against Gram-positive organisms and the Gram-negative cocci and has therefore a range very similar to that of penicillin. Polymyxin is active against the Gram-negative bacilli including the Ps. pyocyaneus. As there is no antagonistic action between the two antibiotics, they together cover the whole range of pyogenic organisms commonly causing eye infection.

Both bacitracin and polymyxin are suitable for topical application but have disadvantages when given systemically. Bacitracin is highly nephrotoxic (Finland and Wright, 1953), and save in exceptional circumstances 
is unsuitable for systemic therapy. Polymyxin is less toxic and may be given systemically if used with caution, but is however liable to produce neurotoxic side-effects (Kagan and others, 1951). As there is now such a wide choice of antibiotics, it is good practice to use those which are toxic when given systemically for topical application wherever possible, reserving those more suitable for systemic therapy for this purpose. Susceptible organisms rarely develop resistance to polymyxin either in vivo or in vitro (Jawetz and Coleman, 1949; Pulaski and others, 1949). Bacterial resistance to bacitracin can be increased in vitro by successive subculture of the organism in media containing sublethal concentrations of the antibiotic, but resistance is developed with difficulty (Gezon and others, 1950).

Ointment containing bacitracin and polymyxin has been used in the treatment of skin infections. Kile and others (1953) reported 459 cases, most of which responded well to the treatment. They used ointment consisting of 400 units bacitracin and 10,000 units polymyxin "B " per gramme of petrolatum. This anhydrous base ensured stability. An identical ointment was used in the trials described below.

\section{Use of Polymyxin-Bacitracin Ointment in Ophthalmology \\ Bacteriological Properties}

A simple qualitative illustration of the range of activity in vitro of ointments containing bacitracin, polymyxin, and a mixture of the two is given in Figs 1-3.

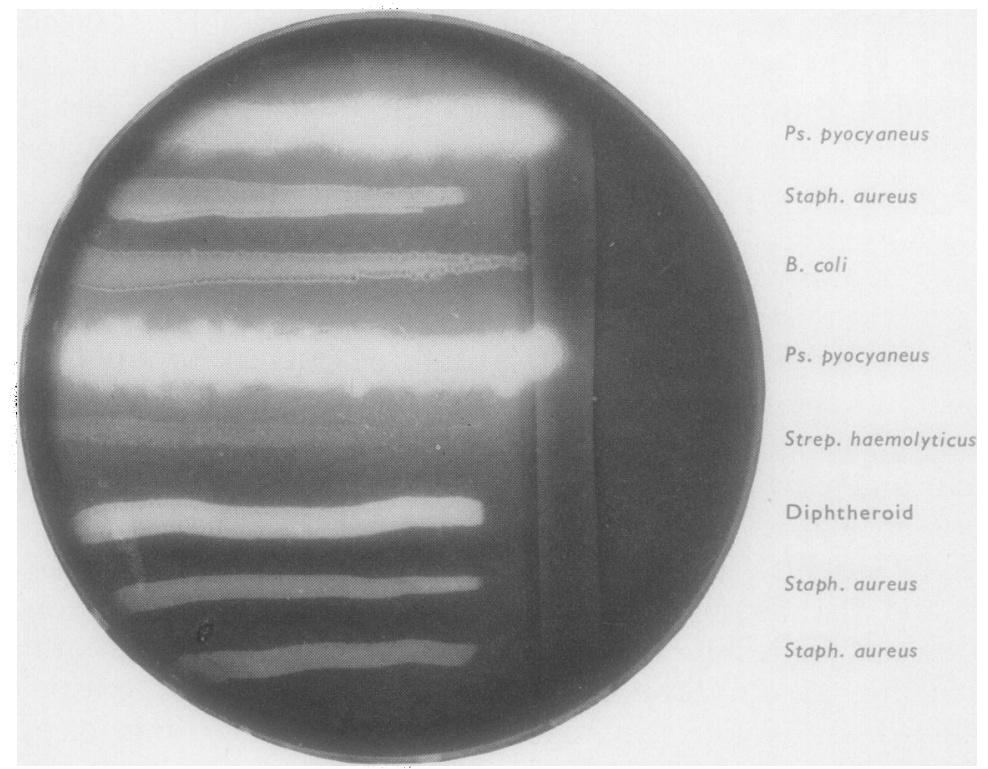

FIG. 1.-Bacitracin ointment, $\mathbf{4 0 0}$ units/g. Growths of Gram-positive organisms, staphylocci, streptococci, and diphtheroids inhibited. Gram-negative bacilli $P s$. pyocyaneus and B. coli not inhibited. The two strains of Ps. pyocyaneus have even invaded the groove containing bacitracin. 


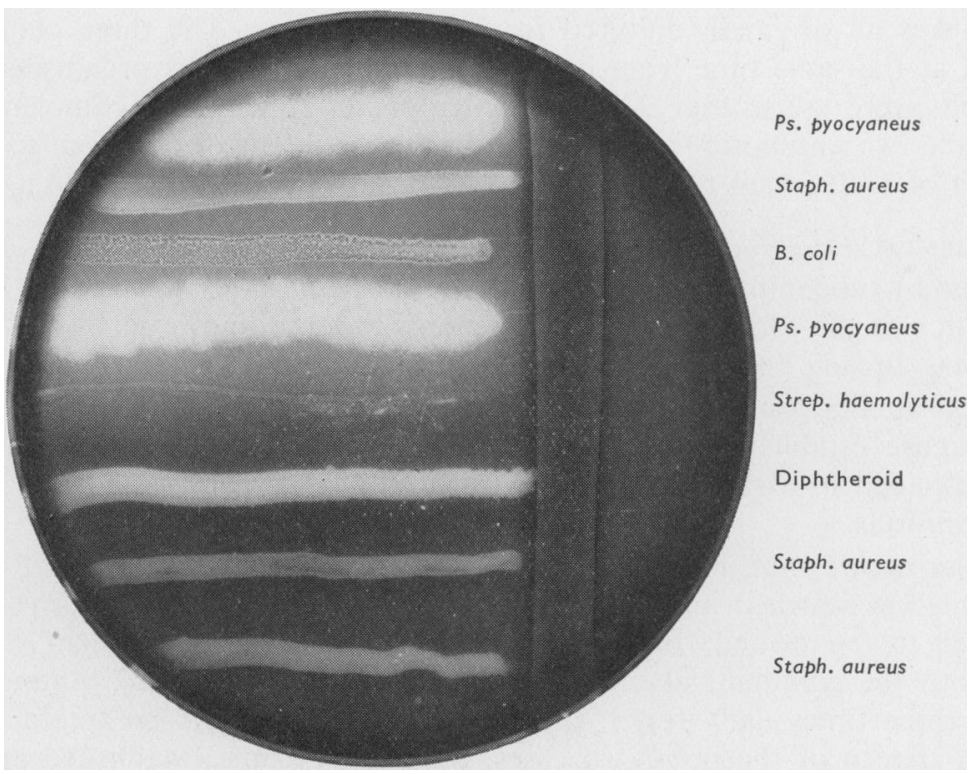

Fig. 2.-Polymyxin "B" ointment, 10,000 units/g. Growths of Gram-positive organisms not inhibited or inhibited to a very small extent. Gram-negative bacilli inhibited.

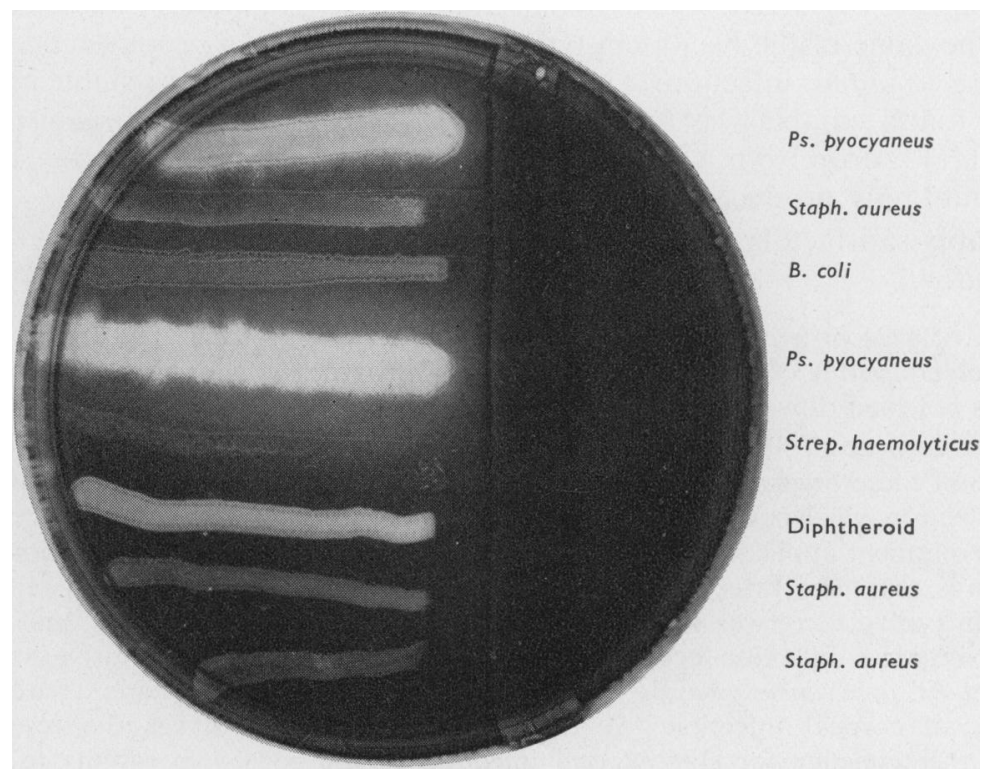

FIG. 3.-Bacitracin-polymyxin " B" ointment, 400 and 10,000 units/g. Growths of both Gram-positive organisms and Gram-negative bacilli inhibited. 
In each case the ointment was applied to a groove in an agar plate seeded with test organisms all originally cultured from eye infections. The three plates were inoculated at the same time from broth cultures of the various organisms. The photographs were taken after $24 \mathrm{hrs}$ incubation at $39^{\circ} \mathrm{C}$. The complementary nature of the two antibiotics is well shown in Fig. 3; the mixture is seen to inhibit the growth of both Gram-positive and Gram-negative organisms.

Experimental Ps. Pyocyaneus Infection of the CoRnea.-The value of polymyxin given by subconjunctival injection in the treatment of established Ps. pyocyaneus infection of the cornea, both experimentally induced and occurring in clinical practice, has already been demonstrated (Ainslie and Smith, 1952; Ainslie, 1953).

The local application of an ointment to the conjunctival sac could not be expected to cure established infection due to so virulent an organism. It was, however, decided to assess its efficacy as a prophylactic against the infection of corneal abrasions.

A circular area $5 \mathrm{~mm}$. in diameter in the centre of the right cornea of each of forty rabbits was scarified, and a drop from a $24-\mathrm{hr}$ broth culutre of Ps. pyocyaneus was applied to the wound. In 23 cases polymyxin-bacitracin ointment was then instilled into the conjunctival sac and the lids were closed. The ointment was reapplied three times daily for 3 days. In seventeen rabbits no treatment was given. In sixteen of the untreated cases, infection became established and progressed rapidly to involve the whole cornea in from 24 to 48 hours. Of the treated cases fifteen remained uninfected, slight infection which cleared within 3 days occurred in six, and in two infection was severe and continued to progress despite treatment.

EXPERIMENTAL STAPH YLOCOCCUS AUREUS INFECTION.-An attempt was then madeo to assess the value of the bacitracin-polymyxin ointment as a prophylactic against Staphylococcus aureus infection. It was impossible to produce a reliable infection in the way found suitable when using Ps. pyocyaneus as the infecting organism. The application of a drop from a 24-hr broth culture of Staph. aureus to the scarified cornea would only produce infection in about 25 per cent. of cases.

Reasonably satisfactory infection could, however, be established by the following method:

$0.03 \mathrm{ml}$. of a $24 \mathrm{hr}$ broth culture of Staph. aureus was injected intracorneally. This raised a bleb consisting of the superficial layers of the cornea. The raised portion of the cornea was scarified down to the encysted fluid.

The right corneae of 46 rabbits were injected and scarified; 21 were left as controls, and sixteen of these became heavily infected, the remainder showing either no infection or slight infection which subsided spontaneously; 25 corneae were treated with bacitracinpolymyxin ointment applied to the conjunctival sac after the corneal scarification. The ointment was reapplied three times daily for 3 days. In nine instances there was no infection, in twelve there was slight infection subsiding within 2 to 3 days, and in four marked progressive infection occurred. The results were not as conclusive as in the series using Ps. pyocyaneus, mainly because of the difficulty of producing a satisfactory staphylococcal corneal infection. A further difficulty which makes the results less definite is the tendency for staphyloccal infection of the cornea in rabbits to resolve spontaneously. Infection in the treated cases was, however, significantly less than in the untreated. 


\section{Clinical Trials}

Blepharitis.-In 33 cases of ulcerative blepharitis, bacitracin-polymyxin ointment was applied to the lids four times daily for the first week of treatment and then twice daily for a further 2 to 3 weeks. In addition, saline lotion was used to help in the removal of crusts and discharge from the lid margins.

In 24 patients symptoms and signs disappeared completely. They have remained symptom-free for from 3 to 6 months without further treatment. In six cases recurrence followed the cessation of treatment but further treatment has kept the condition under control. One case improved initially and then relapsed, and further therapy brought no benefit. Two cases did not respond at all.

Before treatment was started cultures were taken from the lid margins in each case. Staphylococcus aureus was grown from seventeen cases, KochWeeks bacillus from three, Staphyloccus albus from three, B. xerosis from three, and $B$. coli from one; no growth was obtained in six instances. Of the two cases which failed to improve, one had produced a growth of Staph. aureus and one had produced no growth. The persistently recurrent case had produced a growth of $B$. xerosis only. Of the six other cases which recurred but nevertheless responded to further treatment, four had given a growth of Staph. aureus, one of B. xerosis, and the other no growth.

Corneal Ulceration.-Twenty cases of non-specific corneal ulceration were treated with bacitracin-polymyxin ointment applied twice daily, and in addition atropine drops were instilled daily in all but two cases. Healing occurred rapidly in every case, the period of treatment required being from 3 to 8 days. No ulcers of specific aetiology such as dendritic ulcers were included in this series.

Corneal Injury.-In 123 cases of corneal injury due to embedded foreign body ( 88 cases) and abrasions ( 35 cases) the ointment was used as a prophylaxis against infection. An application to the conjunctival sac was made at the patient's first attendance. Twice daily applications were made until healing was complete, which took from 1 to 8 days. In the more severe cases atropine drops were applied in addition.

Of the 123 cases so treated, 115 healed uneventfully; in eight a moderate degree of corneal infiltration occurred but settled within a few days; in the remaining five infiltration was more severe and carbolization was required. After this procedure and continuous treatment with the ointment healing was rapid. Nebula formation was minimal in all cases.

In the total number of cases treated (176) there was no allergic response, though in nine instances the patients complained of slight irritation after applying the ointment. Five of the cases of blepharitis treated had given a history of an allergic response to previous treatments but showed no such response when treated with polymyxin and bacitracin. 
Conclusions.-It is not claimed that the results of this series of cases indicate that bacitracin-polymyxin ointment applied locally to the eye is necessarily superior to other forms of antibiotic therapy. The results show, however, that it is a practical method of treatment and that when used empirically good results are to be expected in the treatment of superficial ocular infections and in the prophylaxis against wound infection. Furthermore, use is made of two antibiotics essentially suitable for local application even though they are not entirely satisfactory when given parenterally. Those antibiotics with a very low toxicity when given systemically could well be reserved for this method of administration.

\section{SUMMARY}

(1) An antibiotic ointment containing bacitracin and polymyxin suitable for ophthalmic use is described.

(2) The range of activity of the ointment covers all pyogenic organisms likely to infect the eye.

(3) Increase in resistance of sensitive organisms to polymyxin and to bacitracin rarely occurs.

(4) The ointment is non-irritant when applied to the eyelids and conjunctival sac and no allergic response occurred in any of the cases treated.

(5) It is suggested that antibiotics such as bacitracin and polymyxin should be used for local application wherever possible allowing antibiotics more suitable for systemic therapy to be reserved for this purpose.

(6) The ointment has been found effective in the treatment of blepharitis and corneal ulceration. It also appears to be useful as a prophylactic agent against ocular wound infection.

My thanks are due to the surgeons of the Moorfields and Westminster Central Eye Hospital who referred cases to me for treatment, and to Sir Stewart Duke-Elder for his assistance and advice. It is a pleasure to acknowledge the help of Dr. Charles Smith of the Institute of Ophthalmology who prepared the plates depicted in Figs 1-3.

The photographs were taken by the Medical Illustration Department at the Institute of Ophthalmology.

\section{REFERENCES}

AINSLIE, D. (1953). British Journal of Ophthalmology, 37, 336.

and SMITH, C. (1952). Ibid., 36, 352.

Cameron, E. H. (1949). Ibid., 33, 368.

Finland, M., and Wright, S. S. (1953). Practitioner (Lond.), 171, 660.

Gezon, H. M., Fasan, D. M., and Collins, G. R. (1950). Proc. Soc. exp. Biol. (N. Y.), 74, 505.

JAWetZ, E., and Coleman, V. R. (1949). J. Lab. clin. Med., 34, 751.

KaGAN, B. M., KReVSKy, D., Milzer, A., and LoCKe, M. (1951). I Ibid., 37, 402.

KIle, R. L., RockWell, E., and Schwarz, J. (1953). Arch. Derm. Syfh. (Chicago), 68, 296.

Pulaski, E. J., Baker, H. J., Rosenburg, M. L., and Connell, J. F., JR. (1949). J. clin. Invest., 28, 1028. 